\title{
Opioids and seizures
}

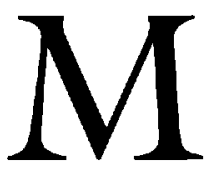

ANY anaesthetic and analgesic agents have been reported to cause seizure-like activity in patients. ${ }^{1,2}$ Epileptic seizures are the clinical manifestations (signs and symptoms) of excessive abnormal activity of neurons in the cerebral cortex. ${ }^{3}$ Epileptic seizures have electrophysiological correlates (epileptiform discharges) that usually, but not always, can be recorded by a scalp electroencephalogram (EEG). Epileptic EEG activity may arise from neurons in either the cortical or subcortical brain regions. Epileptic phenomena cannot always be clearly distinguished from a variety of nonepileptic symptoms produced by abnormal neuronal activity in subcortical structures. Myoclonus is a nonspecific term used to define certain quick involuntary muscle jerks. When cortically generated, they are referred to as epileptic myoclonus. Other forms of myoclonus reflect subcortical mechanisms with no EEG changes (nonepileptic myoclonus) and are more closely related to movement disorders or other neurological symptoms. Because not all forms of epileptic seizures are associated with electrical discharges that can be detected by scalp electrodes, depth or epidural electrodes may be needed. Usually, the diagnosis of an epileptic seizure needs the simultaneous observations of both behavioural and EEG abnormalities. Factors that may determine whether an anaesthetic agent actually produces epileptic seizures or not, include the description of the clinical and EEG evidence of an epileptic seizure, the type of EEG monitoring performed and also the patient population studied.

Opioids are one group of anaesthetic agents that have frequently been reported as causing "seizures." Opioid administration may result in neuroexcitation, ranging from nystagmus, extremity flexion, rigidity, myoclonus and seizures. ${ }^{4}$ The neuroexcitatory phenomena produced by opioids are not fully understood. Changes in central catecholamine concentrations in the dopaminergic pathways have been proposed as possible explanations. Other suggested mechanisms include opioid induced increases in glutamate-activated currents or an increase in the release of excitatory neurotransmitters. EEG epileptic seizures have been reported in response to morphine, meperidine, fentanyl and alfentanil in animal studies. ${ }^{5-7}$ Also opioid induced EEG seizure activity has been noted in nonepileptic patients following chronic oral or intramuscular meperidine administration, attributed to its metabolite, normeperidine. ${ }^{4}$

The numerous reports describing seizure-like activity after opioids (fentanyl, sufentanil and alfentanil) have been well reviewed ${ }^{1,8-10}$ Most of these reports failed to have an EEG or, if they did, no epileptic seizure activity was detected. In many reports, if no EEG was used intraoperatively, patients had postoperative EEG investigations and yet no evidence of epileptic activity was found. Most of these studies were performed in patients who did not have any history of epilepsy. Many of these patients did have abnormal motor activity, myoclonus and/or rigidity. Three explanations have been proposed to explain the nature of opioid induced muscle activity. ${ }^{10}$ The first explanation is that opioids may block cortical inhibitory pathways, thus allowing lower centres to express excitability resulting in clonus. It has been postulated that larger concentrations of opioids may fail to induce myoclonus because such concentrations are likely to block both higher and lower centres of motor control. ${ }^{8}$ The second explanation suggests that opioid induced motor activity represents a form of exaggerated muscle rigidity that may sometimes resemble seizures. ${ }^{11}$ Thirdly, opioid induced abnormal motor activity may represent subcortical seizures, which are unlikely to be detected by surface EEG recordings. ${ }^{6}$

Smith $e t$ al. attempted to answer the question of whether opioid induced rigidity represented epileptic seizure activity. ${ }^{9}$ They studied 127 patients, anaesthetized with large doses of fentanyl, sufentanil and alfentanil. EEG recordings were performed with six surface electrodes, though, in four patients intranasal electrodes were added to look for subcortical activity. Patients also had clinical assessment of movements and rigidity as well as haemodynamic monitoring. Clinically, 46 patients manifested intense rigidity resembling seizures, severe stiffness of limbs and trunk with myoclonic movements and nystagmus. The only observed EEG activity that could be interpreted as epileptiform consisted of small sharp waves related to muscle activity or artifact. Otherwise, there was no EEG indication of epileptic seizure activity in any of the cortical or subcortical tracings. As well, there were no signs 
of sympathetic activity during the episodes of rigidity or myoclonic movement. Despite the evidence from their study, the authors do make the comment that they do not have absolute evidence that none of the patients experience epileptic seizures. Epileptic seizure activity could have occurred in deeper structures, such as the hippocampus or amygdala, but these would not be detected by cortical electrodes. Subcortical electrodes in four patients was not a large enough series. Also, intranasal electrodes are less sensitive than depth or epidural electrodes in detecting epileptic seizure activity from the medial temporal lobe structures and hippocampal area.

Most studies and case reports have been in patients who were not known to have epilepsy. There has been one study by Templehoff $e t$ al. looking at the effect of fentanyl in nine patients undergoing surgery for intractable complex partial seizures. ${ }^{12}$ During induction of general anaesthesia with moderate to high dose fentanyl, continuous recordings with electrocorticographic electrodes were taken from both temporal lobes and hippocampal gyrus. Eight patients exhibited epileptic seizure activity. This activity was detected in the perihippocampal leads. An interesting finding from this study was that fentanyl induced epileptic seizure activity from the contralateral healthy temporal lobe occurred in four patients. Thus, opioids may have the potential to induce epileptic seizure activity in nonepileptic patients in areas that are poorly accessible to monitoring with surface electrodes.

Cascino $e t a l$. reported a retrospective study investigating the effect of alfentanil on the electrocorticography in 23 patients during surgery for intractable seizures under general anaesthesia. ${ }^{13}$ Subdural electrodes were placed on the lateral temporal surface and depth electrodes into the amygdala and hippocampus. They found that $50 \mu \mathrm{g} \cdot \mathrm{kg}^{-1}$ alfentanil $i p$ increased the frequency of epileptiform discharges in 20 patients. One patient had a recorded electrographic seizure.

In rats, EEG recordings from the perihippocampal structures, which contain a high density of opioid receptors, have shown epileptic seizure activity area during the administration of fentanyl and alfentanil. ${ }^{5,7}$ In contrast to other previous human studies and reports, Tempelhoff and Cascino both found activation of epileptiform activity to opioids. ${ }^{12,13}$ Some differences may help to explain the ability to document epileptiform activity in these two studies. Electrocorticographic tracings with multiple electrode placement, especially from the hippocampal area which allowed for optimal assessment of the temporal distribution of electrical activity of the brain, were used. The other major difference is that all the patients in these two studies had documented epilepsy and were having surgery for this disorder.
The question of whether opioids induce epileptic seizures in nonepileptic patients remains uncertain but, probably the evidence indicates that there is no reason not to use opioids in the general population. Perhaps, some caution is indicated in patients with documented history of epilepsy, but further investigations are needed. Interesting, and a potentially useful consideration, is the use of opioids, especially alfentanil, during electrocorticographic recordings to help define the epileptic focus in patients undergoing surgery for epilepsy under general anaesthesia.

\section{Les morphiniques sont-ils épileptogènes?}

Plusieurs anesthésiques et analgésiques sont considérés comme des proconvulsivants. ${ }^{1,2}$ Les crises épileptiformes, par ailleurs, sont les manifestations cliniques (signes et symptômes) d'une activité neuronale anormale au niveau du cortex cérébral. ${ }^{3}$ Les crises épileptiques ont une corrélation électrophysiologique, les décharges épileptiformes, qu'il est possible, la plupart du temps, d'enregistrer sur le cuir chevelu par l'électroencéphalogramme (ÉEG). L'activité ÉEG épileptique peut se manifester dans une région corticale ou sous-corticale. Il est parfois difficile de faire la distinction entre des phénomènes épileptiques et une variété de symptômes non épileptiques produits par une activité neuronale sous-corticale anormale. Le terme non spécifique de myoclonie est employé pour décrire certaines secousses musculaires involontaires répétitives. Si elles sont d'origine corticale, on les appelle myoclonies épileptiques. D'autres formes de myoclonie reflètent des mécanismes sous-corticaux qui ne modifient pas l'ÉEG (myoclonie non épileptique) et sont en relation étroite avec des désordres moteurs ou d'autres symptômes neurologiques. Comme toutes les formes de crises épileptiformes ne sont pas associées à des décharges électriques détectables par des électrodes placées sur le cuir chevelu, il peut devenir nécessaire d'insérer des électrodes profondes ou épidurales. Pour pouvoir diagnostiquer une crise d'épilepsie, il faut avoir observé des anomalies simultanées du comportement et de l'ÉEG. La description de la crise et ses manifestations électroencéphalographiques, le type d'ÉEG réalisé ainsi que les sujets étudiés constituent les facteurs déterminants de l'épileptogénécité d'un anesthésique. 
Les morphiniques font partie d'une classe d'agents anesthésiques fréquemment considérée comme épileptogène. ${ }^{1}$ L'administration d'un morphinique peut entraîner de la neuroexcitation, dont les manifestations sont aussi variées que le nystagmus, la flexion des extrémités, la rigidité, les myoclonies et les convulsions. ${ }^{4}$ On connaît mal pourquoi les morphiniques sont neurotoxiques. Comme explication, on a proposé des altérations des concentrations centrales des catécholamines situées au niveau des voies de conduction dopaminergiques. D'autres mécanismes ont été aussi été suggérés dont l'intensification induite par les morphiniques de courants activés par le glutamate et l'augmentation de la libération des neurotransmetteurs de l'excitation. Chez l'animal, on a enregistré sur l'ÉEG des crises épileptiques après l'administration de morphine, de mépéridine, de fentanyl et à d'alfentanil. ${ }^{5-7}$ Chez des patients non épileptique, l'administration orale ou intramusculaire prolongée de mépéridine a provoqué de l'activité épileptoïde qu'on a attribuée à son métabolite, la normépéridine.

Les nombreux comptes rendus de crises épileptoïdes consécutives à l'administration de morphiniques (fentanyl, sufentanil, alfentanil) ont été passés en revue. ${ }^{1,8-10}$ Dans la plupart des cas, l'ÉEG est absent, ou s'il est présent, il ne met pas en évidence d'activité épileptiques. Dans plusieurs des interventions réalisées sans monitorage électroencéphalographique, l'investigation ÉEG postopératoire ne démontrait pas non plus d'activité épileptiforme. La plupart de ces études portaient sur des patients sans passé épileptique. Plusieurs d'entre eux présentaient une activité motrice anormale, identique à la myoclonie et/ou la rigidité. Trois hypothèses sont proposées pour expliquer la nature de l'activité musculaire induite par les morphiniques. ${ }^{10}$ La première énonce que les morphiniques peuvent bloquer les voies de conduction inhibitrices, permettant ainsi aux centres inférieurs de manifester une activité clonique. On a postulé que des concentrations plus importantes de morphiniques ne provoqueraient pas de myoclonie parce qu'elles pourraient bloquer à la fois les centres supérieurs et inférieurs du contrôle de la motricité. ${ }^{8} \mathrm{La}$ deuxième explication suggère que l'activité motrice induite par les morphiniques représente en réalité une forme de rigidité musculaire qui ressemble parfois à des convulsions. ${ }^{11}$ Troisièmement, l'activité motrice anormale provoquée par les morphiniques peut représenter des crises épileptoïdes sous-corticales qu'il est pratiquement impossible de déceler avec des électrodes d'enregistrement ÉEG superficielles. ${ }^{6}$

Smith et al. ont essayé de préciser si la rigidité induite par les morphiniques correspondait à des crises épileptiformes. ${ }^{9}$ Ils ont étudié 127 patients anesthésiés avec de fortes doses de fentanyl, de sufentanil et d'alfentanil. Ils ont enregistré l'ÉEG avec six électrodes de surface, mais ils ont en plus inséré chez quatre patients des électrodes intranasales dans le but d'explorer l'activité sous-corticale. Après la mise en marche du monitorage hémodynamique, ils ont recherché systématiquement chez leurs patients les mouvements anormaux et la rigidité. Quarante-six patients ont présenté une rigidité épileptoïde très intense, avec une raideur des membres et du tronc accompagnée de mouvements myocloniques et de nystagmus. À l'interprétation de l'ÉEG, la seule activité épileptoïde consistait en de petites pointes ondes reflétant une activité musculaire ou des artefacts. Par ailleurs, l'enregistrement cortical et sous-cortical de l'ÉEG ne révélait aucune activité épileptique. Malgré ces résultats, les auteurs affirment ne posséder aucune preuve absolue permettant d'affirmer qu'un seul parmi ces patients avait présenté une crise épileptiforme. Si une activité épileptiforme était survenue dans des structures plus profondes comme l'hippocampe et l'amygdale, il était impossible de la détecter avec des électrodes corticales. De plus, l'enregistrement obtenu chez quatre patients porteurs d'électrodes sous-corticales ne pouvait représenter une démonstration valide. Les électrodes intranasales sont moins sensibles que les électrodes profondes ou épidurales pour montrer les crises épileptiformes provenant des structures médianes du lobe temporal et de l'hippocampe.

La plupart des études et des observations provenaient de patients sans passé épileptique. Toutefois, une étude par Templehoff $e t$ al. s'est intéressée aux effets du fentanyl chez neuf sujets opérés pour des convulsions réfractaires partielles et complexes. ${ }^{12}$ Pendant l'induction de l'anesthésie avec des doses modérées à fortes de fentanyl, ils ont réalisé de façon continue des enregistrements aux lobes temporaux et à la circonvolution de l'hippocampe. Huit patients ont présenté de l'activité épileptique. Cette activité provenait des électrodes placées à l'hippocampe. Il est intéressant de noter que, dans cette étude, l'administration de fentanyl a aussi provoqué une activité épileptiforme au niveau du lobe temporal controlatéral sain chez quatre patients. Parce que certaines régions sont difficilement accessibles au monitorage avec des électrodes superficielles, on ne peut donc exclure que les morphiniques puissent induire des crises épileptiformes chez des patients non épileptiques.

Une étude prospective électrocorticographique réalisée par Cascino et al. portait sur 23 opérés pour des crises convulsives réfractaires sous anesthésie générale. ${ }^{13}$ Des électrodes sous-durales étaient placées sur la surface temporale latérale et profondément dans l'amygdale et 
l'hippocampe. Ils ont trouvé que l'alfentanil $50 \mu \mathrm{g} \cdot \mathrm{kg}^{-1}$ $i v$ augmentait la fréquence des décharges épileptiformes chez 20 patients. Un patient a fait une crise qu'ils ont enregistrée électrographiquement.

Chez le rat, les enregistrements ÉEG de structures situées en périphérie de l'hippocampe, qui contiennent une haute densité de récepteurs morphiniques, ont montré une zone d'activité épileptiforme pendant l'administration de fentanyl et d'alfentanil. ${ }^{5,7}$ Contrairement à d'autres études et observations antérieures chez l'homme, Templehoff et Cascino ont tous deux démontré que les morphiniques stimulaient l'activité épileptiforme. ${ }^{12,13}$ Dans ces deux études, quelques différences méthodologiques peuvent s'avérer utiles pour comprendre comment l'activité épileptiforme a été documentée. D'abord, les enregistrements électrocorticographiques à plusieurs électrodes, spécialement dans la région de l'hippocampe, étaient utilisés pour évaluer de façon optimale la distribution temporale de l'activité électrique cérébrale. L'autre différence majeure consistait en ce que les patients de ces deux études étaient reconnus comme épileptiques et étaient spécifiquement opérés pour cette affection.

Il n'est pas sûr que les morphiniques provoquent des crises épileptiformes chez des patients qui ne sont pas épileptiques, mais nous n'ayons aucune raison valable pour pas les utiliser de façon habituelle. La prudence pourrait être de mise chez les patients dont l'épilepsie est prouvée, mais des études plus approfondies sont nécessaires. L'utilisation des morphiniques et spécialement de l'alfentanil pendant les enregistrements électrocorticographiques présente beaucoup d'intérêt et pourrait être utilisé avec avantage pour identifier les foyers épileptogènes pendant les interventions pour épilepsie sous anesthésie générale.

\section{References}

1 Modica PA, Tempelhoff $R$, White PF. Pro-and anticonvulsant effects of anesthetics. (Part I) Anesth Analg 1990; 70: 303-15.

2 Modica PA, Tempelhoff R, White PF. Pro-and anticonvulsant effects of anesthetics. (Part II) Anesth Analg $1990 ; 70: 433-44$.

3 Engel J Jr. Seizures and Epilepsy. Philadelphia: F.A. Davis Company, 1989: 3-5.

4 Bailey PL, Stanley TH. Intravenous opioid anesthetics. In: Miller RD (Ed.). Anesthesia, 4th ed. New York: Churchill Livingston Inc., 1994: 4.

5 Carlsson C, Smith DS, Keykhah MM, Engelbach I, Harp $J R$. The effects of high-dose fentanyl on cerebral circulation and metabolism in rats. Anesthesiology 1982; 57: $375-80$.
6 Tommasino C, Maekawa T, Shapiro HM, KeiferGoodman J, Koblenberg RW. Fentanyl-induced seizures activate subcortical brain metabolism. Anesthesiology 1984; 60: 283-90.

7 Kofke WA, Garman RH, Tom WC, Rose ME, Hawkins $R A$. Alfentanil-induced hypermetabolism, seizure, and histopathology in rat brain. Anesth Analg 1992: 75 : 953-64.

8 Scott JC, Sarnquist FH. Seizure-like movements during a fentanyl infusion with absence of seizure activity in a simultaneous EEG recording. Anesthesiology 1985; 62: $812-4$

9 Smith NT, Bentbuysen JL, Bickford RG, et al. Seizures during opioid anesthetic induction - are they opioidinduced rigidity? Anesthesiology 1989; 71: 852-62.

10 Sprung J, Schedewie HK. Apparent focal motor seizure with a Jacksonian march induced by fentanyl: a case report and review of the literature. J Clin Anesth 1992; 4: $139-43$.

11 Bentbuysen JL, Smith NT, Sanford TJ, Head N, DecSilver $H$. Physiology of alfentanil-induced rigidity. Anesthesiology 1986; 64; 440-6.

12 Tempelboff R, Modica PA, Bernardo KL, Edwards I. Fentanyl-induced electrocorticographic seizures in patients with complex partial epilepsy. J Neurosurg 1992; 77: 201-8.

13 Cascino GD, So EL, Sharbrough FW, et al. Alfentanilinduced epileptiform activity in patients with partial epilepsy. J Clin Neurophysiology 1993; 10: 520-5. 